\title{
Developing Personalized E-Books: A Multi-Layered Approach
}

\author{
Konstantina Chatzara, Athanasios Mpantsos, Demosthenes Stamatis \\ and Athanasios Tsadiras \\ Department of Informatics \\ Technological Educational Institute of Thessaloniki \\ P.O.BOX 14561, 54101 Thessaloniki, Greece \\ Email: tsadiras@it.teithe.gr
}

\begin{abstract}
Users of hypermedia systems are heterogeneous, came from different backgrounds and have different level of knowledge in the use of information systems. This paper discusses a multi-layered approach for developing personalized e-books. The layers regard both Human Computer Interaction/Navigation level and the Content of the e-book. These layers are defined and described in detail. The tei-Book architecture is also presented in the paper and it is proposed as suitable for providing personalized e-book views to the various e-book readers. This is done by an E-Book manager that manage to provide the appropriate e-book view according to the User Profiles and Book Profile Components that dynamically are developed by the tei-Book. Future work includes the evaluation of the personalized e-book views and also the evaluation of system's usability.
\end{abstract}

\section{Introduction}

Adapting books to electronic form in order to be readable by users in a computer system is a concept that motivated research very early. The book metaphor has acted as the primary way of presenting e-learning systems in a book like lay out [1]. Electronic browsing systems as SuperBook [2] and the Book Emulator [3], as well as recent commercial offerings of hand held devices of book size [4] are a few of the examples of the research into the e-book domain.

The demands and expectations of diverse users have grown fast. A recent study reports that even for experienced users of common personal computers, approximately $45 \%$ of their time is wasted with frustrating experiences tied to confusing menus, indecipherable dialog boxes, and hard to find functions [5]. The users find themselves disorientated, feeling stressed out and disappointed, end up giving up the learning procedure to avoid further annoyance.

Please use the following format when citing this chapter:

Chatzara, Konstantina, Mpantsos, Athanasios, Stamatis, Demosthenes, Tsadiras, Athanasios, 2006, in IFIP International Federation for Information Processing, Volume204, Artificial Intelligence Applications and Innovations, eds. Maglogiannis, I., Karpouzis, K., Bramer, M., (Boston: Springer), pp. $425-432$ 
The e-book as a learning platform should be able to accommodate for all users but usually it is designed in a unified way, cutters for the middle line of users, excluding the novice ones or makes the application unattractive for the experienced users. The inequity of access to hypermedia products, often called the digital divide [6] addresses the need of adapting the way the user accesses an e learning system to cater for each individual separately.

In order to cater to different user needs, information systems can be tailored manually by the user or automatically by the system. Systems that allow the user to change certain system parameters, and adapt their behavior accordingly, are called adaptable. Systems that adapt to users automatically based on their assumptions about them are called adaptive [7]. Whereas the first group of adaptations aims at enabling and improving the overall access to the information system, the second group of adaptations aims at individualizing one specific hypermedia system.

Personalization of the e learning systems is increasingly important. Research has shown that most users prefer the control afforded by an adaptable approach to personalization rather than a system-controlled adaptive approach. No study, however, has compared the efficiency of the two approaches.

Multi layers have been introduced in order to divide the interface of an application into sections and make each section accessible by the user separately $[8,9,10]$. Instead of presenting all features at once it is proposed to allow the user to choose the features he prefers according to his skills and personal needs. An example of a multi-layered application has been illustrated in the search engines on the Internet in which the novice users just type a keyword in a search box and the results are presented in a certain sequence. Users are usually satisfied with the whole procedure, which is easily accessed. Experienced users might use a more advanced interface, which allows them to use more criteria (country they are seeking information from, time depended results, order of the results when presented). The above approach gives users control over the sets of features available at any moment but a clear separation between the different functions is incorporated. The two layers of accessing the information is presented clearly leaving the user in control.

Each level can be designed separately; nevertheless all layers have to share common aesthetics. Distinct goals that will be achieved in each level have to be clarified. It is vital for the user to navigate through the system without the notion of disorientation. Confusion between switching between layers has to be avoided.

Cognitive learning models have to be incorporated in order to define the principles of the design of a multi layered e-learning application and usability testing as well as user feedback will be essential. Facilities as help menu that clarifies the use and scope of each level have to be examined and the interaction between interface layers and layers of the content presentation also have to be addressed.

By adding to this debate the issue of not only adapting the navigation but adapting the content of the subject the e-learning application is designed for, we are presented with a much more complex system. In the following chapter the layers structure of presentation of the content and the navigation will be described, incorporating both the adaptive and the adaptable approach. 


\section{E-Book layers definition}

This paper discusses a multi-layer approach to developing personalized e-books [11]. A system for supporting this personalization process, tei-Book (transformable electronic and intelligent Book) was developed at the Department of Informatics of the Technological Educational Institute (TEI) of Thessaloniki. As it is shown in figure 1, our layered approach, proposes the existence of layers both to the HCI (human-computer interaction/navigation level) of the e-book and to the content of the e-book.

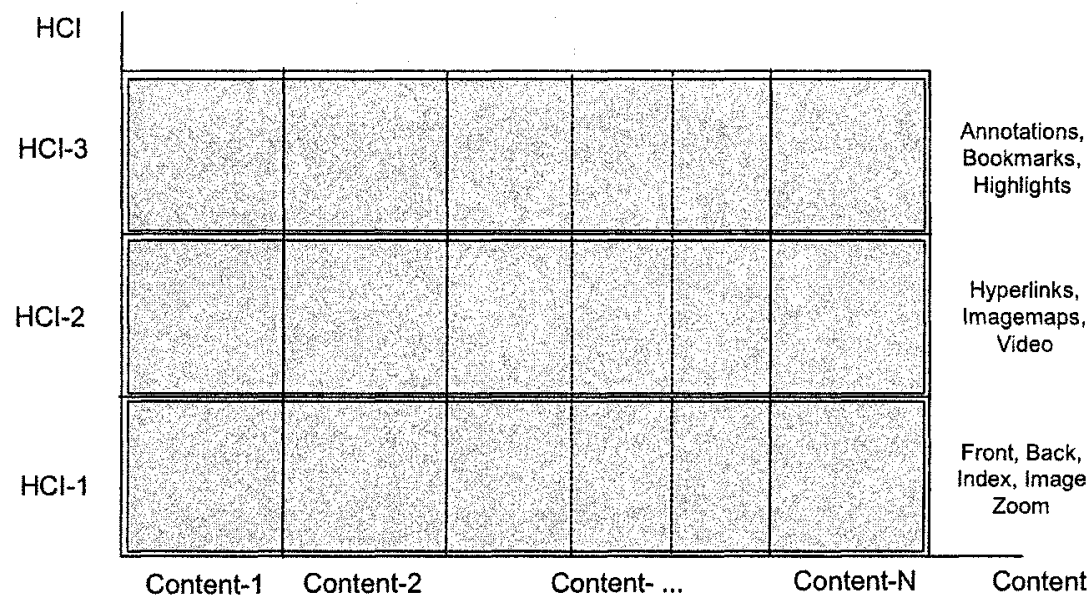

Fig. 1. Layers concerning both to the $\mathrm{HCI}$ of the e-book and the content of the e-book.

According to the proposed design, there are three different layers/levels of HCI. These are the following:

Level HCI-1. This layer is designed for first-time and novice users of computer systems, having no experience of how to use hypermedia. In this layer, only a static menu will be presented, having simple functions such as return to index, go to next and previous page and exit (see figure 2). Additional to these, images can zoom-in to reveal details of various initially small images in the e-book. The user of this layer will use the book metaphor and he will be able to navigate through e-book contents without feeling disoriented.

Level HCI-2. In this level, users that have some experience in hypermedia, additionally to the features of level HCI-1, will be able to find in the e-book, (a) hyperlinks, (b) imagemaps (images are separate in different areas, each area having a link to a corresponding e-book position) and (c) videos with the necessary navigation buttons (play, pause, stop, fast forward, rewind). The user of this level will feel free to navigate more flexibly to the whole book in a much faster manner. 


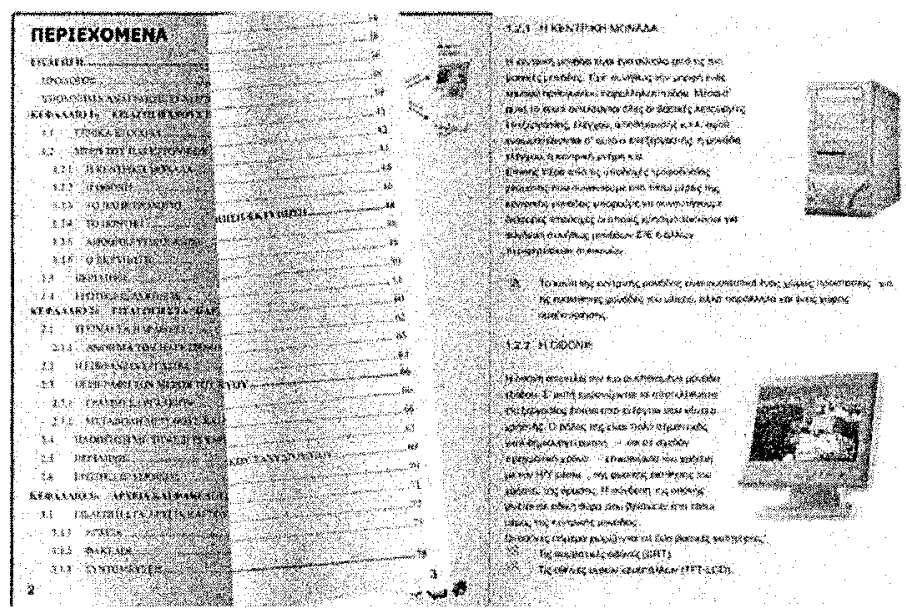

Fig. 2. Tei-Book with book like layout, page turning simulation, table of contents, front and back page cover

Level HCI-3. In this top level, additionally to the features of level HCI-2, the user will be able to personalize the e-book, by (a) adding his own bookmarks on the pages of the e-books, (these work as hyperlinks to specific e-book positions), (b) highlighting sentences that he considers important for him, and (c) adding personal annotations on the pages of the e-book, that can be his personal comments on ebook's content (Figure 3). Every time the user of level 3 exits the e-book, information regarding the personalized version of the e-book will be saved. In this way the user will be able to load his own personalized version of the e-book and continue his study from the point he stopped studying the e-book, viewing also his past annotations, highlights and bookmarks.

Separating out the HCI-layers enable users to learn HCI features in a meaningful sequence, while help screens are avoided. Nevertheless help menu can be added to explain the functions of each $\mathrm{HCl}$ level. E-book readers can gain confidence and after mastering layer HCI-1 move on to higher layers, when needed or when they had time to experiment with.

The e-book can also have different levels of content. The e-book domain expert can identify different levels of users according to their past knowledge on the e-book subject, their age and the depth of the knowledge they are looking for in the e-book. In this way, level Content-1 will correspond to the simplest content version which will suitable for the absolute beginner of the e-book subject, having no prerequisites. In the same manner, highest content level, level Content- $N$, will correspond to the most advanced content version of the e-book that will be suitable for the most experienced on the subject and concerned reader of the e-book. The number of different content levels is not defined, since it depends on the subject and the scope of the readers the e-book's authors want to reach. 


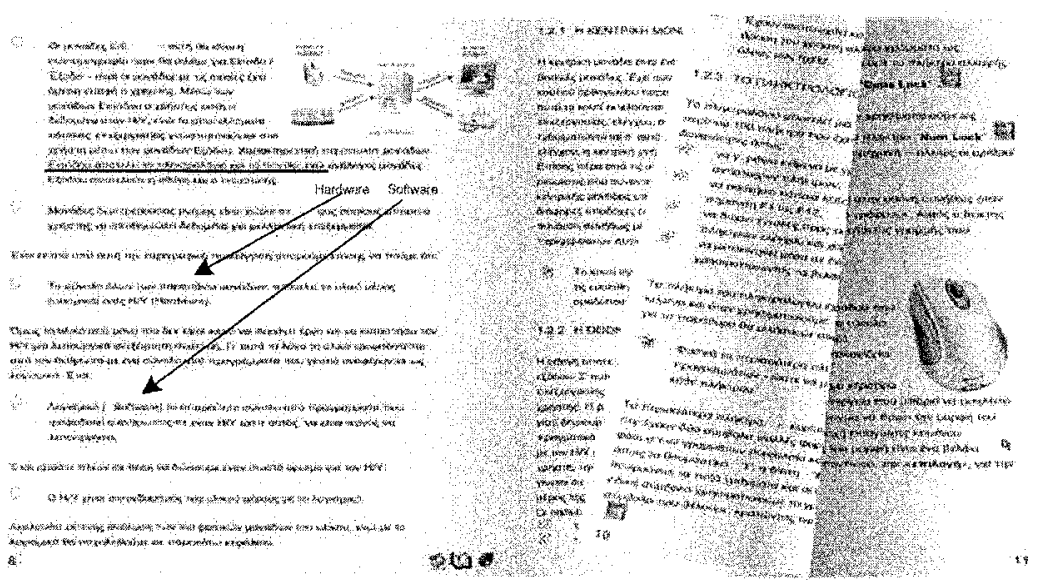

Fig. 3. Tei-Book with user's personal annotations and highlighted content.

According the above and figure 1 , an e-book reader who is novice to the use of hypermedia but very experienced on the e-book subject, should use the e-book tuned to levels $\mathrm{HCI}-1$ \& Content-N, whereas an experienced hypermedia user with no background knowledge on the subject of the e-book, should use the e-book tuned to levels HCI-3 \& Content-1.

\section{The tei-Book Architecture}

The personalization supported by the tei-Book system that we propose, is orthogonal in the sense that HCI layered based personalization and content based personalization could be applied as two separated processes. These processes can then be combined to provide the final view of personalized e-book to its users.

Figure 4 shows the architecture of the tei-Book system. The content material constituting a specific e-book is stored in the e-book Content Base.

The HCI Adaptation module is the one responsible to decide for the HCI layer adaptation pertinent to each user. In order to do so it keeps a data base of user profiles. These profiles are updated when the user initiates a reading session, either based on his direct will or by following an interactive questionnaire approach in order to diagnose his level. In such a way the e-book is functioning as an adaptable hypermedia system. The profiles could also be updated automatically by the system which monitors the user interaction through a microadaptation submodule $(\mathrm{HCl}$ Monitor) in order to allow for dynamic HCI layer switching. In such a way the ebook could be considered as an adaptable hypermedia system.

The Content Adaptation module is responsible to decide for the content layer adaptation which provides different views to different users. In order to do so it keeps a data base of book profile components. These book profile components are updated: 


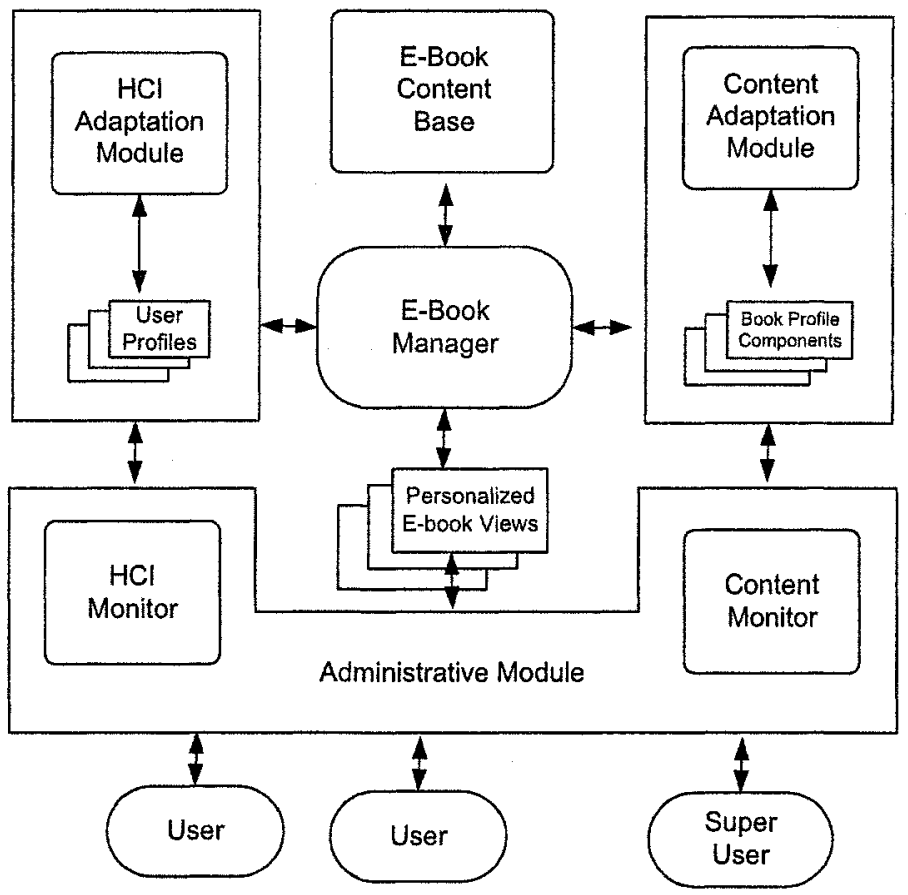

Fig. 4. The tei-Book architecture

(a) When a user initiates a reading session at $\mathrm{HCI}$ level 3, where content adaptations tools (for bookmarking, annotation, paragraph hide et.c.) are provided. In this case, a book profile consisting of a single book profile component is created which governs content personalization for the user in question.

(b) When a special user (e.g. author of the e-book or experienced teacher using the e-book as e-learning material for its students) initiates a reading session in order to transform the e-book content. In this case, a discrete number of book profile components could be generated corresponding to different levels of content adaptation. The special user then has the possibility of assigning these levels to specific user groups. Based on these content profile components the content adaptation module can produce one or more book profiles to be assigned to final users.

In both cases, user's actions regarding content change are controlled through the Content Monitor Module. In the second case content based personalization acts as a tool to a teacher to annotate and transform the e-book in order to enhance a specific strategy for reading to be followed by the students.

The e-book Manager subsystem is responsible for producing the personalized ebook views for the specific users. It does so by using the e-book content base and by collaborating with the $\mathrm{HCl}$ and Content Adaptation modules. By providing sufficient redundancy and separation of content from HCI layout, the system ensures through 
the e-book Manager module that for any given situation adaptation is possible without involving the complete content management chain.

The Administrative module apart from accommodating the $\mathrm{HCl}$ and Content Monitors is responsible for controlling the whole user interface to the e-book and for keeping user accounts. The system works as a web based client server application and users need to go through a login authentication procedure in order to work with their personalized version of the e-book. Additionally when a personalized e-book view is finalized it can be compiled and used off-line by its user as a stand alone hypermedia document.

\section{Conclusions - Future Work}

This paper suggested a multi-layer approach to developing personalized e-books. Two types of personalization are possible: $\mathrm{HCI}$ based layers of personalization and content based layers of personalization. These two types could either be applied separately or could be combined. The layers were defined and the architecture of the tei-Book system, which is used as a framework for developing personalized e-books, was presented. A first version of the tei-Book was implemented together with a complete e-book case study, an e-book containing an introduction to Computer Systems. This case study will be used in order to evaluate the personalized e-book views that are produced during the interaction between users (both readers and teacher) and the tei-book system. Based on the evaluation results a more complete version of the suggested architecture will be implemented. Usability testing is also essential and comparison between the adaptable and the adaptive aspects of personalization has to be examined in the future.

\section{References}

1. Golovchinsky, G., Marshall, C.: Designing Electronic Books, ACM Transactions on Computer-Human Interaction, Vol. 7, No. 2, (2000)

2. Egan, D. E., Remde, J.R., Gomez, J.M., Landauer, T.K., Eberhardt, J., and Lochbaum, C.C. Formative designevaluation of SuperBook. ACM Transactions on Information Systems, (1989)

3. Benest, I.D. A hypertext system with controlled hype. In McAleese, R. and Green, C. (eds.). Hypertext: State of the Art. Intellect Books, Oxford, 1990.

4. D. Berleant, : Models for reader interaction systems, Ninth International Conference on Information and Knowledge Management (CIKM), Washington, D.C., ACM Press, (2000), pp 127-133.

5. Ceaparu, I., Lazar, J., Bessiere, K., Robinson, J., and Shneiderman, B., Determining causes and severity of end-user frustration, International Journal of Human-Computer Interaction, (2004).

6. Shneiderman, B., Promoting universal usability with multi-layer interface design, ACM Conference on Universal Usability, ACM Press, New York (2003)

7. Fink, J., Kobsa, A., Schreck, J.: Personalized Hypermedia Information Provision through 
Adaptive and Adaptable System Features: User Modeling, Privacy and Security Issues, Proc. of the Workshop Adaptive Systems and User Modeling on the World Wide Web of the 6th lnt. Conf. on User Modeling, Chia Laguna, Sardinia (1997)

8. Plaisant, C., Kang, H., and Shneiderman, B., Helping users get started with visual interfaces: multi-layered interfaces, integrated initial guidance and video demonstrations, Proc.Human-Computer Interaction International, Lawrence Erlbaum Associates,

9. Kang, H., Plaisant, C., and Shneiderman, B., New approaches to help users get started with visual interfaces: Multi-layered interfaces and Integrated Initial Guidance, Proc. of the Digital Government Research Conference, Boston, MA (May 2003).

10. Dunlop, MD, Johnson, CW, Reid, J.: Exploring the Layers of Information Retrieval Evaluation. In Interacting with Computers, 10 (3), (1998), p. 225-236

11. Chi, E. H.; Hong, L.; Heiser, J.; Card, S. K.: eBooks with indexes that reorganize conceptually. Extended Abstracts of the ACM International Conference on Human Factors in Computing Systems (CHI 2004); Austria, (2004), 1223-1226. 